\title{
Erratum to: Homogeneous Self-cleaning Coating on Cellulose Materials Derived from TIP/TiO 2 P25
}

The following erratum relates to the article:

"Homogeneous Self-cleaning Coating on Cellulose Materials Derived from TIP/TiO 2 P25" by

Nika Vero, Silvo Hribernik, Patrizia Andreozzi, and Majda Sfiligoj-Smole, which has been published online in Fibers and Polymers, DOI 10.1007/s12221-010-0716-2.

The publisher apologizes for an error in one of the author names: Nika Vero should be Nika Veronoski. 